\title{
MAGNETS FOR THE CERN PS BOOSTER TRANSFER LINE
}

\author{
George S. Clark, Alan J. Otter, and Paul Reeve, TRIUMF 4004 Wesbrook Mall, Vancouver, B.C., \\ Canada V6T 2A3
}

\begin{abstract}
TRIUMF is building 22 magnets for the CERN PS Booster Transfer Line as part of the Large Hadron Collider (LHC) upgrade. These magnets replace similar existing magnets in the transfer line but have higher fields as the maximum proton energy is being increased from $1 \mathrm{GeV}$ to $1.4 \mathrm{GeV}$. The magnets, 12 quadrupoles and 10 dipoles, are laminated for pulsed operation with a 1.2 second cycle time. The original magnets operated DC. The new magnets must fit within the existing space envelopes and mate to existing support stands.
\end{abstract}

front end region. The lower BVT10 bender supports two DVT dipoles and a second BVT10 bender. The upper bender is cantilevered.

\section{THE BENDERS}

The DVT magnets [1] are small window frame correction dipoles with the field oriented horizontally to give a vertical bend. To fit the magnet into the allowed space the coil leads had to exit from inside the coil. This coupled with the CERN requirement that the coils are splice free, forced the coil manufacturer to wind the

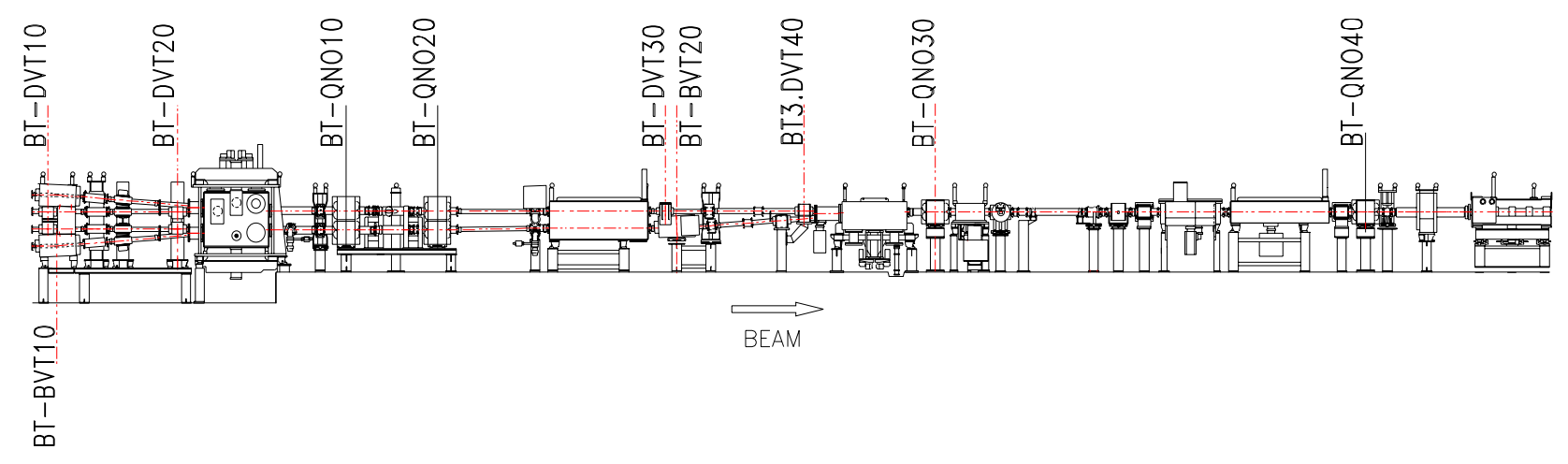

Figure 1 The CERN PS Booster Transfer Line

\section{INTRODUCTION}

TRIUMF is participating in a collaboration with CERN to upgrade the Large Hadron Collider. As part of this project TRIUMF is supplying 12 quadrupole and 10 dipole magnets. These magnets are to replace existing magnets in transfer lines connected to CERN's PS Booster. The new magnets must fit within the space envelope of the original magnets despite the maximum proton energy increasing from 1 to $1.4 \mathrm{GeV}$. The change from DC to pulsed operation with 1.2 second cycle time required the magnets be laminated.

Figure 1 shows the PS Booster Transfer Line and the locations where TRIUMF supplied magnets are to be installed.

The first four dipoles in the Booster Transfer Line will be supplied by TRIUMF. One side of this front end region is very close to the Booster so that space is extremely tight. Figure 2 shows the magnets of the
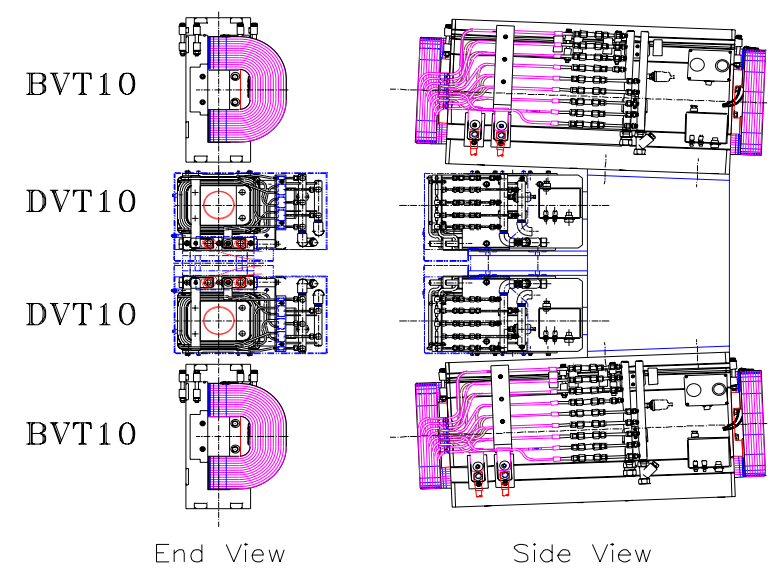

Figure 2 Front End Region

saddle shaped coils from the outside-in. TRIUMF is supplying five magnets for installation and two spares.

TRIUMF is supplying the two BVT10 benders [2,3] in the front end and a single BVT20 bender [2,3] further down the transfer line. These window frame dipoles share a common coil design. 
Table 1 Bender Parameters

\begin{tabular}{|l|l|l|l|l|l|l|l|l|l|}
\hline Type & Overall & Effective & Aperture & DC & Field & Bend & Turns & Magnet & Magnet \\
\hline & Length & Length & & Current at & at & Angle & Per & Resistance & Inductance \\
\hline & & & & $1.4 \mathrm{GeV}$ & $1.4 \mathrm{GeV}$ & $1.4 \mathrm{Gev}$ & Coil & & \\
\hline & $\mathrm{m}$ & $\mathrm{m}$ & $\mathrm{m}$ & $\mathrm{A}$ & $\mathrm{T}$ & Radian & & Ohms & H. \\
\hline & & & & & & & & & \\
\hline BVT & 0.4 & 0.383 & 0.102 & 248 & 0.191 & 0.01 & 32 & 0.1 & 0.003 \\
\hline BVT10 & 0.98 & 0.908 & 0.062 & 273 & 0.608 & 0.077 & 112 & 0.24 & 0.043 \\
\hline
\end{tabular}

Table 2 Quadrupole Parameters

\begin{tabular}{|l|l|l|l|l|l|l|l|l|l|}
\hline Type & Overall & Effective & Aperture & Max. & DC & Gradient & Turns & Magnet & Magnet \\
\hline & Length & Length & & Energy & Current at & at & Per & Resistance & Inductance \\
\hline & & & & & Max E & Max E & Coil & & \\
\hline & $\mathrm{m}$ & $\mathrm{m}$ & $\mathrm{m}$ & $\mathrm{eV}$ & $\mathrm{A}$ & $\mathrm{T} / \mathrm{m}$ & & Ohms & $\mathrm{H}$. \\
\hline & & & & & & & & & \\
\hline BI & 0.57 & 0.462 & 0.150 & & 17 & 1.79 & 159 & 0.83 & \\
\hline BT & 0.6 & 0.466 & 0.150 & $1.4 \mathrm{G}$ & 258 & 6.57 & 57 & 0.16 & 0.041 \\
\hline
\end{tabular}

Stresses imposed by lifting and supporting the magnets was not a problem for the original thick yoked BVT benders. The lifting and stand attachment points of the original magnets had to be reproduced in the new laminated magnets. For the BVT magnets these attachment points are not at stress minimizing locations, so considerable work was put into the mechanical design [4,5]. Thick tension straps recessed into the magnet were positioned to accept the attachment points. The thickness of the weld attaching the tension strap to the laminations varies along the length of the magnets.

Table 1 shows the bending magnet parameters.

\section{THE QUADRUPOLES}

TRIUMF is supplying twelve quadrupole magnets in three configurations that share a common steel design. These magnets are a figure-of-eight design [6,7]. Five air-cooled BI-Quadrupoles have been measured at CERN and installed in the Linac Transfer Line leading to the PS Booster. At $15 \mathrm{~A}$ the measured gradient is $1.077 \mathrm{~T} / \mathrm{m}$ and the measured effective length is 0.4598 m.[8].

Seven water cooled BT-Quadrupole magnets are currently under construction. Four of the BT-Quads will be assembled in stacked pairs, one at top the other. The position of the apertures in the pairs is held to a $0.1 \mathrm{~mm}$ tolerance. TRIUMF is now field mapping three of the BT quadrupoles.

Table 2 shows the quadrupole magnet parameters.

\section{REFERENCES}

[1] Alan Otter, "A Review of CERN DVT Dipole Magnets', TRIUMF Design Note TRI-DN-96-5, April 1996.

[2] Alan Otter, 'CERN BV Benders Upgraded for 1.4 GeV Pulsed Operation', TRIUMF Design Note TRIDN-95-32, November 1995.

[3] Alan Otter, 'CERN BV Benders -- OPERA-2D Calculations', TRIUMF Design Note TRI-DN-96-1, February 1996.

[4] G.S.Clark, 'Mechanical Calculations for the TRIUMF/CERN BV1 Bender', TRIUMF Design Note TRI-DN-96-20, September 1996.

[5] G.S.Clark, 'Mechanical Calculations for the TRIUMF/CERN BV2 Bender', TRIUMF Design Note TRI-DN-96-25, November 1996

[6] Paul A. Reeve, 'Design of BT/BI Quadrupole for CERN Booster Beam Lines', TRIUMF/University of Victoria Design Note VPN-95-3, December 1995

[7] G.S.Clark, 'Mechanical Stress Calculations for the BT/BI Quadrupole for CERN', TRIUMF Design Note TRI-DN-96-4, April 1996

[8] R. Chritin, D. Cornuet, and G. Patron, 'Mesures magnetiques en courant continu d'aimants quadripoles de type BI', CERN Note Technique SLMS/Note 97-15, Fevrier 1997 